\title{
The Multiple Regression Analysis of the Influence Factors of Foreigner's Computer Chinese Application Level
}

\author{
Yali Mu and Hao Bai* \\ Beijing Language and Culture University, Beijing, 100083, China \\ *baihao@blcu.edu.cn
}

Keywords: Foreign students; Computer application level; Influencing factors

Abstract. In this study, 27 foreign students studying in the basic course of computer application are taken as the research object. The application level of computer is taken as the dependent variable. The mastery of computer terminology, production and demonstration of PPT, input speed, Correct rate of Chinese characters and Chinese level as independent variables. By means of multiple regression analysis and hierarchical regression analysis, the factors that influence the application level of computer are investigated. Finally, all the above-mentioned factors affect the students' computer application level. Among them, the mastery of computerized terminology has a strong predictive effect on the computer application level in Chinese.

Acknowledgements: This article is funded by the school-level new project of Beijing Language and Culture University in 2017. The project number is: C180102.

\section{来华留学生计算机汉语应用水平影响因素的多重}

\section{回归分析}

\author{
穆雅丽，白浩 \\ 北京语言大学汉语进修学院，北京 100083
}

摘要：本研究以选修计算机应用基础这门课的 27 名来华留学生为研究对象, 将其计算机的汉语应用水平作为因变量, 将计算机专业术语掌握程度、PPT 的制作与演示、汉字录入速度、汉字录入正确率、汉语水平作为自变量。采取多重回归分 析与层次回归分析的方法, 对于影响计算机汉语应用水平的因素进行考察, 最终显示: 以上因素均在不同程度上影响留学生 的计算机汉语应用水平，其中，计算机专业术语的掌握对计算机的汉语应用水平有较强预测作用。

关键词: 留学生; 计算机汉语应用水平; 影响因素

\section{1 引言}

针对非计算机专业的留学生，各大高校普遍开设计算机应用基础课(1)无论是作为必修课程还是选修课 程，均受到了来华留学生青睐。

但已有研究表明，计算机应用基础课程存在着计算机基础水平差别巨大、汉语语言的掌握程度不一、 课程通过率较低的问题（沈湘芸等 2009; 林龙心等 2010)。针对这种教学现状, 有学者指出: 引进国内外 优秀教材或自制教材、分班分组授课、专业词汇与专业技能相结合、加强课堂管理、因材施教等教学方法 (白浩, 2014; 蔡晓丽, 2017)。至于具体的实施方法, 要根据学生的兴趣与已有的计算机水平进行有针

${ }^{(1)}$ 本文只探讨针对非计算机专业的留学生开设的计算机基础课程, 即以 office 办公软件的基本应用（word、exce1、PPT 的 基本应用) 为主要内容的基础课程。 
对性的教学（肇恒宇等 2008; 李宇耀，2014）。

综上所述, 目前学者对于留学生计算机应用基础课的现状、存在的问题及解决方法进行了大量的定性 分析。遗憾的是, 对于计算机汉语应用水平的影响因素, 未见实证研究。本文拟通过实验, 从计算机专业 术语掌握程度、PPT 的制作与演示、汉字打字速度、汉字打字正确率以及汉语水平五个角度考察影响留学 生计算机汉语应用水平的因素，为计算机基础课程的分班教学提供可操作性指导。

\section{2 实验研究}

\section{1 实验假设}

实验假设 1: 计算机专业术语 (如, “复制”、“粘贴”、“分栏”)、汉字录入速度、汉字录入正确率、PPT 的制作与演示四个方面均能反应计算机汉语应用水平。

实验假设 2: 汉语水平是影响计算机汉语应用水平的关键因素。

\section{2 操作性定义}

（1）计算机汉语应用水平: 将留学生计算机应用基础课的期末成绩(2作为衡量标准（以下简称 JS）。

（2）汉语水平：计算机应用基础是中级（上）的选修课，我们将 27 名留学生由初级（下）升入中级 （上）的汉语综合课的期末试卷成绩作为衡量其汉语水平的标准（简称 HS）。

（3）计算机专业术语水平：以计算机专业术语的小考成绩作为其衡量标准（简称 SS）。

（4）PPT 的制作与演示：以 PPT 制作与演示的小考成绩作为其衡量标准（简称 PS）。

（5）文字录入速度：以金山打字软件记录的 10 篇文章的平均速度作为衡量标准（WS）。

(6) 文字录入正确率: 以金山打字软件记录的 10 篇文章录入的平均正确率作为衡量标准 (简称 WZ)。

\section{3 研究对象与研究方法}

共有 27 名留学生参加本实验 (其中 12 名女生, 15 名男生, 平均年龄 25 岁), 均为北京语言大学汉语 进修学院中级（上）选修计算机应用基础这门课（34 学时）的学生。

以 JS 为因变量，SS、PS、WS、WZ、HS 为自变量。首先，对 5 个自变量与 1 个因变量进行 person 相关 分析, 初步探讨各自变量与因变量 JS 的关系, 以及各自变量之间的关系。再进行多重回归分析, 以 JS 为 因变量, 其他 5 个变量为自变量, 建立多重回归模型。以上均使用 SPSS20.0 进行统计分析。

\section{3 结果分析}

本文有效数据为 26 名留学生的 JS、SS、PS、WS、WZ、HS 数据, 由于 1 名男生缺勤而导致的小考成绩 不全, 故将该名学生的全部数据剔除。

\footnotetext{
(2) 计算机课期末试卷=计算机专业术语常识*30\%+word 文章录入并编辑*40\%+PPT 简单制作*30\%, 考试时间 120 分钟。
} 


\section{1 计算机汉语应用水平与其预设的影响变量的相关分析结果}

表 1 计算机汉语应用水平及其预设影响变量的相关 $(r)$

\begin{tabular}{lllllll}
\hline 变量 & JS & SS & PS & WS & WZ & HS \\
\hline JS & 1.000 & & & & & \\
SS & $0.713 * *$ & 1.000 & & & & \\
PS & $0.437 *$ & $0.452 * *$ & 1.000 & & & \\
WS & $0.431 *$ & 0.372 & 0.246 & 1.000 & & \\
WZ & 0.042 & 0.074 & -0.101 & 0.324 & 1.000 & \\
HS & $0.532 *$ & $0.620 * *$ & $0.710 * *$ & 0.228 & -0.146 & 1.000 \\
\hline
\end{tabular}

注: * $\mathrm{P}<0.05, * * \mathrm{P}<0.01$

相关分析表明, JS 与 $\mathrm{SS}$ 相关最高 $(r=0.713, \mathrm{P}<0.01)$, HS 次之 $(r=0.532, \mathrm{P}<0.05)$, 然后是 $\mathrm{PS}(\mathrm{r}=0.437$, $\mathrm{P}<0.05)$ 。最后是 WS $(r=0.431, \mathrm{P}<0.05)$ 。JS 与 $W Z$ 未见相关 $(r=0.042 ， \mathrm{P}>0.05)$ 。(3)

再看预设变量之间的相关程度： PS 与 $S S$ 的相关最高（ $r=0.452 ， P<0.01 ） ; S S$ 与 WZ 之间未见相关 $(r=0.074, P>0.05)$ 。而这四项知识应用技能与 HS 均存在相关。可见, 预设自变量之间存在着复杂的相 关关系。下面我们将利用多重回归分析做进一步的研究。

\section{2 计算机汉语应用水平与其预设的影响变量的多重回归分析结果}

利用多重回归分析的进入法, 将自变量全部进入建立的回归方程中，汇总得到表 2 。

表 2 留学生计算机汉语应用水平影响因素的多重回归分析

\begin{tabular}{|c|c|c|c|c|c|c|c|c|c|}
\hline \multirow{3}{*}{$\begin{array}{l}\text { 因 变 } \\
\text { 量 }\end{array}$} & \multirow{3}{*}{ 预测变量 } & \multicolumn{2}{|c|}{ 非标准化回归数 } & \multicolumn{6}{|c|}{ 标准化偏 } \\
\hline & & \multirow{2}{*}{\multicolumn{2}{|c|}{$\beta$ 标准误 }} & 回归数 & $\mathrm{t}$ & P & $\mathrm{R} 2$ & $\mathrm{~F}$ & P \\
\hline & & & & Beta 值 & & & & & \\
\hline \multirow[t]{6}{*}{ JS } & 常量 & \multicolumn{2}{|c|}{49.28895 .331} & & .517 & .611 & .558 & 5.044 & .004 \\
\hline & SS & .276 & .099 & .557 & 2.783 & .011 & & & \\
\hline & PS & .119 & .345 & .073 & .344 & .735 & & & \\
\hline & WS & .789 & .670 & .202 & 1.178 & .253 & & & \\
\hline & WZ & -.268 & .973 & -.405 & -.276 & .785 & & & \\
\hline & HS & 068 & 258 & .082 & .334 & .742 & & & \\
\hline
\end{tabular}

型经方差检验, $F(5,21)=5.044, P=0.004$, 即回归方程是有意义的。并且 $R 2=0.558$, 即表明模型 中引入的五个变量可解释影响计算机汉语应用水平总变异的 $55.8 \%$, 见表 2 。

由表 2 可以看出, 只有 SS 对 JS 的影响显著 $(\mathrm{P}<0.05)$, 其他变量对 JS 的影响均不显著。但由于 SS 与 HS 存在很高相关 $(r=0.620, P<0.01)$, 无法排除是否由于样本数量的原因, 致使 HS 对 JS 的影响不显 著。因此, 再采用层次回归的方法单独考察 SS 以及 HS 对 JS 的影响。

\footnotetext{
(3) 根据科恩 (1988) 对相关系数大小的相关指导: 绝对值处于 $0.1-0.29$, 相关系数小; 绝对值处于 $0.3-0.49$, 相关系数中; 绝对值处于 $0.5-1.0$ 之间, 相关系数大。
} 


\section{3 计算机汉语应用水平的预设变量的层次回归分析}

表 3 计算机术语掌握、汉语水平对计算机汉语应用水平的的层次回归分析

\begin{tabular}{|c|c|c|c|c|c|c|c|}
\hline \multirow[b]{2}{*}{ 模型 } & \multirow[b]{2}{*}{$\mathrm{R}$} & \multirow[b]{2}{*}{$\mathrm{R}$ 方 } & \multirow[b]{2}{*}{ 调整 R 方 } & \multirow{2}{*}{$\begin{array}{c}\text { 标准估计 } \\
\text { 的误差 }\end{array}$} & \multicolumn{3}{|c|}{ 更改统计量 } \\
\hline & & & & & $\mathrm{R}$ 方更改 & F 更改 & $\begin{array}{c}\text { Sig. F 更 } \\
\text { 改 }\end{array}$ \\
\hline 1 & $.622 \mathrm{a}$ & .386 & .270 & 11.367 & .386 & 3.306 & .030 \\
\hline 2 & $.747 \mathrm{~b}$ & .558 & .447 & 9.889 & .171 & 7.747 & .011 \\
\hline 3 & $.745 c$ & .555 & .471 & 9.677 & .555 & 6.555 & .001 \\
\hline 4 & $.747 \mathrm{~d}$ & .558 & .447 & 9.889 & .002 & .112 & .742 \\
\hline
\end{tabular}
a. 预测变量：(常量), HS, WZ, WS, PS.
b. 预测变量：(常量), HS, WZ, WS, PS, SS.
c. 预测变量：(常量), SS, WZ, PS, WS.
d. 预测变量：(常量)，JS，WZ，PS，WS，HS.

如表 3, 我们单独考察两个变量对 JS 的影响。将除 SS 以外的四个变量先期输入模型, 即得到表中的 模型 1 , 控制了其他变量的影响, 最后 SS 输入模型时, 得到模型 2 , 发现其对 JS 的贡献仍达到 $17.1 \%(\Delta \mathrm{R} 2$ $=0.171)$, 其贡献是统计上差异显著的 $(\mathrm{P}=0.011)$ 。同理, 考察 HS 这个单独的变量对 JS 的贡献, 发现有 $0.2 \%(\Delta R 2=0.002)$ 且差异并不显著 $(P=0.742)$ 。

综上所述，实验假设 1 成立，SS、PS、WS 、WZ 、HS 均能反应 JS。其中，SS 最能反应 JS，具有很 高的贡献度。实验假设 2 不成立, HS 的高低并不是 JS 的关键因素。

\section{4 综合讨论}

\section{1 计算机专业术语与基础应用对计算机汉语应用水平的反应}

计算机汉语应用水平与各自变量的相关程度为: $S S(r=0.713, \mathrm{P}<0.01)>\mathrm{HS}(\mathrm{r}=0.532, \mathrm{P}<0.05)>$ $\mathrm{PS}(\mathrm{r}=0.437, \mathrm{P}<0.05)>\mathrm{WZ}(\mathrm{r}=0.431, \mathrm{P}<0.05)>\mathrm{WS}(\mathrm{r}=0.042, \mathrm{P}>0.05)>$ 。多重回归分析与层次回 归分析结果也均显示, SS 对 JS 具有无可替代的贡献度，最能体现 JS。

为何计算机专业术语的掌握程度最能体现计算机汉语应用水平呢？我们认为诸如“复制、粘贴、分栏、 首字下沉” 等计算机专业术语, 是留学生学好计算机基础应用操作技能的前提。试想, 如果留学生连题目 要求, 比如进行 Word 编辑时 “首字下沉” 都看不懂, 或者 Word 操作时工具栏里不认识 “行距” 二字, 那么他就很难拥有很高的计算机汉语应用水平了。

\section{2 汉语水平对计算机汉语应用水平的影响}

汉语水平 (HS) 对计算机汉语应用水平 (JS) 具有较高的相关程度 $(r=0.532, \mathrm{P}<0.05)$, 这似乎证实了 HS 对计 JS 的影响。然而, 通过层次分析发现, HS 对 JS 的单独贡献度并不高 ( $\Delta \mathrm{R} 2=0.002, \mathrm{P}=0.742$ )。因 此, HS 并不是决定 JS 的关键因素。换言之, 汉语水平不高的人, 只要计算机课堂认真学习理论知识, 多 加练习计算机应用技能，同样能取得优异成绩。

那么, HS 与 JS 有较高的相关程度, 作何解释呢? 我们认为, HS 并非直接对 JS 有影响, 而是通过影 响 SS $(r=0.620, P<0.01)$ 与 PS $(r=0.710, P<0.01)$, 间接对 JS 产生影响。汉语水平高, 掌握计算机专 业术语就会变得轻松一些, PPT 里输入的文字段落的内容, 错误就会相应少一些, 同理, 汉语水平高, Word 文字录入时, 速度与正确率相应比较高。 


\section{5 结论}

本文以计算机的汉语应用水平作为因变量, 将计算机专业术语掌握程度、PPT 的制作与演示、汉字录 入速度、汉字录入正确率、汉语水平作为自变量。采取多重回归分析与层次回归分析的方法, 对于影响计 算机汉语应用水平的因素进行考察, 得出结论: 以上因素均能影响留学生的计算机汉语应用水平。其中, 计算机专业术语的掌握有独特的贡献作用, 能很大程度地预测计算机汉语应用水平。因此, 计算机基础应 用的教学, 教师要首先应关注计算机专业术语的讲解, 学生熟练掌握了专业术语, 才是提高计算机汉语应 用水平的前提。

此外, 本文处于探索性研究, 样本容量不高, 相关系数与显著性指标只是在该样本容量基础上所得, 因此上述实验结论, 有待进一步研究验证。

\section{6 致谢}

本文受 2017 年北京语言大学校级新开课项目资助，项目编号为：C180102。

\section{参考文献}

[1] 白浩. 来华留学生预科生计算机基础课教学研究一以北京语言大学为例 [A] //王瑞烽, 王俊毅 《汉语进修教育多 角度研究》, 北京: 中国书籍出版社, 2014 年: 56-65。

[2] 蔡晓丽. 留学生全英文计算机公共课教学质量保障方法探析 [J]. 《福建电脑》，2017 年，第 1 期。

[3] 李宇耀. 语言类外国留学生计算机基础课程教学方法探讨 $[J]$. 《吉林省教育学院学报》, 2014 年(下旬)。

[4] 林龙新, 杜宝荣, 范荣强. 非计算机专业留学生的计算机基础课程教学研究 $[J]$, 《计算机教育》, 2010 年, 第 20 期。

[5] 沈湘芸，李莉平，张建生．东南亚留学生计算机课程教学方法初探 $[J] ， 《$ 成功》（教育）2009 年第 4 期。

[6] 肇恒宇，肖峰，张特来. 医学院校外国留学生计算机教学的研究与探讨 $[J] ， 《$ 电脑知识与技术》，2008 年。

\section{References}

[1] H.Bai: A Study on the Basic Computer Course Teaching of Preppies of International Students in China -Taking Beijing Language and Culture University as an Example[A]// R.F.Wang and J.Y.Wang: A Multi-Angle Study of Chinese Advanced Education, Beijing: Chinese Book Publishing House(2014), P:56-65.(In Chinese)

[2] X.L.Cai: An Analysis of the Teaching Quality Assurance Methods of All English Computer Public Courses for Foreign Students[J], Fujian Computer(2017)No.1.(In Chinese)

[3] Y.Y.Li: Discussion on the Teaching Method of Computer Basic Course for Foreign Students of Language Class[J], Journal of Educational Institute of Jilin Province, 2014(the last ten-day of a month).(In Chinese)

[4] L.X.Lin, B.R.Du and R.Q.Fan: The Computer Basic Course Teaching Resource for Non-Computer Major Overseas Students[J], Computer Education, (2010)No. 20.(In Chinese)

[5] X.Y.Shen, L.P.Li and J.S.Zhang: The Primary Exploration of the Teaching Methods of Computer Courses for Overseas Students in Southeast Asia[J], Success(Education) (2009)No.4.(In Chinese)

[6] H.Y.Zhao, F.Xiao and T.L.Zhang: The Research of Computer Teaching for Foreign Students in Medical University[J], Computer Knowledge and Technology, 2008.(In Chinese) 